\title{
An Effective Linear Precoding Scheme to Resolve High PAPR and High OOBE Problems of OFDM systems
}

Hikaru Kawasaki ( $\sim$ h_kawasaki@nict.go.jp )

NICT https://orcid.org/0000-0002-9176-1969

Takeshi Matsumura

NICT

Fumihide Kojima

NICT

\section{Research Article}

Keywords: 5G, OFDM, linear precoding, out-of-band emission, high peak power

Posted Date: June 1st, 2021

DOI: https://doi.org/10.21203/rs.3.rs-344162/v1

License: (c) (i) This work is licensed under a Creative Commons Attribution 4.0 International License.

Read Full License 


\title{
An Effective Linear Precoding Scheme to Resolve High PAPR and High OOBE Problems of OFDM systems
}

\author{
Hikaru Kawasaki · Takeshi Matsumura . \\ Fumihide Kojima
}

Received: date / Accepted: date

\begin{abstract}
In the fifth-generation of mobile communications, the use of orthogonal frequency-division multiplexing is agreed by the Third Partnership Project as it was in the fourth-generation. On the other hand, the major problems of its transmitted signals are high out-of-band emission (OOBE) that causes strong interference to adjacent frequency bands and high peak power characteristics that limit device costs and power savings. For future communications, this paper proposes an effective method to resolve the problems based on linear precoding with the theoretical analyses. Numerical experiments show that compared with the usual OFDM, the proposed method can achieve $25 \mathrm{~dB}$ lower OOBE, $2 \mathrm{~dB}$ lower peak power, no error rate degradation with a practical computational complexity simultaneously.
\end{abstract}

Keywords 5G, OFDM, linear precoding, out-of-band emission, high peak power

\section{Introduction}

Orthogonal frequency-division multiplexing (OFDM) is widely used thanks to its high transmission rates and robustness to multipath channels along with the implementation ease offered by fast Fourier transform (FFT) algorithm. It is also well matched to multiple-input multiple-output technology. In the fifth-generation (5G) (3GPP, 2018), the third generation partnership project (3GPP) has agreed on the use of cyclic-prefix (CP)-based OFDM (CP-OFDM) and CP-based discrete Fourier transform (DFT) spread OFDM (DFT-s-OFDM) waveforms (Myung et al., 2006), as it was in the fourth-generation (4G) 3GPP standards (3GPP, 2016a). However,

H. Kawasaki, T. Matsumura, and F. Kojima

Wireless Systems Laboratory, Wireless Networks Research Center

National Institute of Information and Communications Technology

3-4 Hikarino-oka, Yokosuka, 239-0847 Kanagawa, Japan

Tel.: +81-46-5050

Fax: +81-46-5059

E-mail: \{h_kawasaki, matsumura, f-kojima $\} @$ nict.go.jp 
in contrast to the advantages, the OFDM scheme has two major problems. One is heavy power fluctuation of its transmitted signals because the waveforms have characteristics similar to Gaussian noise. The fluctuation is a serious constraint on power savings as well as coverage gain, particularly to wireless mobile terminals (Han and Lee, 2005) Another is high out-of-band emission (OOBE) derived from the discontinuity of the adjacent OFDM symbols. The high OOBE causes a serious interference with the adjacent frequency bands (Weiss et al., 2004; Brandes et al., 2006).

Linear precoding is a powerful tool to combat various issues of the OFDM signals by multiplying a well-designed matrix by data symbols prior to the inverse DFT (IDFT) for OFDM modulation. The DFT-s-OFDM is one of the most famous linearly precoded OFDM whose linear precoding is implemented by the DFT matrix. The contribution focuses on reduction of the heavy power fluctuation for mobile terminals implemented with low-cost but low-efficient power amplifiers in $4 \mathrm{G}$ systems based on the 3GPP standards. However, the power spectrum of the DFT-s-OFDM signals is theoretically equivalent to that of the plain OFDM signals with the high OOBE. Meanwhile, the scheme of the linear precoding has also attached a lot of attention with respect to the OOBE suppression (Chung, 2006a,b, 2008; Xu and Chen, 2009; van de Beek and Berggren, 2009; van de Beek, 2009, 2010; Ma et al., 2011; Zheng et al., 2012; Clarkson, 2017; Pitaval et al., 2017; Kawasaki et al., 2018; Mohamad et al., 2018; Hussain et al., 2019; Kawasaki et al., 2019). It is referred to as spectral precoding, and Ref. (Chung, 2008) has first introduced it to the CP-OFDM. In the spectral precoding, the data symbols are precoded to satisfy linear constraints for OOBE suppression. Unlike filtering and windowing techniques (Weiss et al., 2004; Zhang et al., 2018; Mizutani et al., 2019), it ensures that CP length remains effective and thus simplifies the OFDM structure, e.g., the frequency-domain equalizer (FEQ). From these vivid advantages, the spectral precoding would be put into practical like the great success of the conventional DFT-s-OFDM. In particular, spectral-orthogonal (SO) precoding is promising in terms of no BER degradation (Xu and Chen, 2009; van de Beek, 2010; Ma et al., 2011; Clarkson, 2017; Kawasaki et al., 2018, 2019). The computational complexity in the orthogonally precoded OFDM systems is practical by efficiently construction of its precoder compared with the plain OFDM or DFT-s-OFDM systems (Clarkson, 2017; Kawasaki et al., 2018, 2019). However, the power fluctuation mitigation is ignored, computationally expensive, or marginally achieved without fine analyses in most of the SO precoding schemes.

This paper proposes an effective linear precoding scheme to resolve the major problems of the OFDM systems The key advantage of the proposed method compared to the plain OFDM scheme is that

- a strong OOBE suppression by about $25 \mathrm{~dB}$ at band-edges in power spectrum density (PSD)

- a practical reduction of power fluctuation by roughly $2 \mathrm{~dB}$ better in peak-to-average-power ratio (PAPR)

- no degradation in bit error rate (BER)

- the computational amounts for the proposed precoding matching to that of the FFT for the OFDM modulation 
can be simultaneously achieved while each performance is theoretically evaluated. It suggests that the proposed method can resolve the problems of interest with a minimal implantation cost in the conventional OFDM systems already equipped with precoding structure.

The rest of the paper is organized as follows. Section II presents a system model for linearly precoded systems including the DFT-s-OFDM and spectrally precoded OFDM. The proposed method to achieve both PAPR reduction and OOBE suppression are revealed with theoretical analyses in Section III while the performance of the proposed method is also verified by numerical experiments in Section IV. Section IV concludes this paper.

\section{System Model}

This paper considers extensions of the OFDM scheme with respect to linear precoding techniques. As in practical OFDM schemes, the transmitted signal is expressed in block transmission as

$$
s(t)=\sum_{i=0}^{\infty} s_{i}(t-i T)
$$

where $T=T_{\mathrm{S}}+T_{\mathrm{g}}$ is the symbol length of an OFDM symbol with the $\mathrm{CP}, T_{\mathrm{S}}$ is the OFDM symbol duration, $T_{\mathrm{g}}$ is the CP length. Each OFDM symbol $s_{i}(t)$ with the CP is

$$
s_{i}(t)=I(t) \boldsymbol{p}^{T}(t) \overline{\boldsymbol{d}}_{i},
$$

where $\boldsymbol{p}(t)=\left[p_{k_{0}}(t), p_{k_{1}}(t), \ldots, p_{k_{K-1}}(t)\right]^{T} \in \mathbb{C}^{K \times 1}$ with $p_{k}(t)=e^{-j 2 \pi \frac{k}{T_{\mathrm{s}}} t}$ are the subcarriers for the OFDM implemented by the IFFT, $I(t)$ is the window function for the block transmission, $\mathcal{K}=\left\{k_{0}, k_{1}, \ldots, k_{K-1}\right\}$ denotes the subcarrier mapping, $K=|\mathcal{K}|$ is the number of subcarriers, $\overline{\boldsymbol{d}}_{i}=\left[\bar{d}_{i, k_{0}}, \bar{d}_{i, k_{1}}, \ldots, \bar{d}_{i, k_{K-1}}\right]^{T} \in \mathbb{C}^{K \times 1}$ is the modulating symbols for the subcarriers, which is assumed linearly precoded to convey the data symbol $\boldsymbol{d}_{i}=\left[d_{i, 0}, d_{i, 1}, \ldots, d_{i, D-1}\right]^{T} \in \mathbb{C}^{D \times 1}$ containing the $D$ information symbols in some finite constellation. In this paper, $I(t)$ is defined as a rectangular one to keep the effective length of the $\mathrm{CP}$, that is, $I(t)=1$ for $-T_{\mathrm{g}} \leq t<T_{\mathrm{S}}$ while $I(t)=0$ elsewhere, and $\mathbb{E}\left\{\boldsymbol{d}_{i} \boldsymbol{d}_{i}^{\mathrm{H}}\right\}=\boldsymbol{I}_{K}$ is assumed where $\boldsymbol{I}_{l} \in\{0,1\}^{l \times l}$ denotes an identity matrix for an positive integer $l$.

Among linearly precoded OFDM schemes, we are interested in memoryless precoding fashions with the uncorrelation among $\overline{\boldsymbol{d}}_{i}$, that is, $\mathbb{E}\left\{\overline{\boldsymbol{d}}_{i} \overline{\boldsymbol{d}}_{\boldsymbol{i}^{\prime}}^{\mathrm{H}}\right\}=\boldsymbol{O}$ for $i \neq i^{\prime}$. A concise description of memoryless precoding is

$$
\overline{\boldsymbol{d}}_{i}=\boldsymbol{G} \boldsymbol{d}_{i} \in \mathbb{C}^{K \times 1},
$$

where $\boldsymbol{G} \in \mathbb{C}^{K \times D}$ is called precoder. The above formulation is general enough to encompass any linearly precoded OFDM system; the plain OFDM system coincides the case of $D=K$ and $\overline{\boldsymbol{d}}_{i}=\boldsymbol{d}_{i}$. The DFT-s-OFDM is one of the most famous linearly precoded OFDM scheme to mitigate the high PAPR characteristics of the plain OFDM. Its precoder is implemented with $D=K$ as

$$
\boldsymbol{G}=\boldsymbol{W}_{D} \in \mathbb{C}^{D \times D},
$$


where $\boldsymbol{W}_{D}$ is the normalized DFT matrix with the $(m, l)$-th factor defined by $w_{m, l}=$ $e^{j 2 \pi \frac{m}{K} l} / \sqrt{D}$ and can be efficiently implemented by the FFT algorithm. However, the DFT-s-OFDM signals renders the PSD with the same high OOBE as in the plain OFDM counterparts because $\boldsymbol{W}_{D}$ is unitary and thus $\mathbb{E}\left\{\overline{\boldsymbol{d}}_{i} \overline{\boldsymbol{d}}_{i}^{\mathrm{H}}\right\}=\mathbb{E}\left\{\boldsymbol{d}_{i} \boldsymbol{d}_{i}^{\mathrm{H}}\right\}$.

Meanwhile, spectral precoding is a scheme to effectively suppress the OOBE. To suppress the OOBE of the transmitted signal $s(t)$ in (1), it is assumed that linearly independent $M=K-D$ constraints are imposed on $\overline{\boldsymbol{d}}_{i}$ in a homogeneous system $\boldsymbol{A} \overline{\boldsymbol{d}}_{i}=\mathbf{0}$ where $\boldsymbol{A} \in \mathbb{C}^{M \times K}$ is a design parameter. Note that $\boldsymbol{A} \overline{\boldsymbol{d}}_{i}=\boldsymbol{A} \boldsymbol{G} \boldsymbol{d}_{i}=\mathbf{0}_{M}$ for any $i$ reduces to

$$
\boldsymbol{A} \boldsymbol{G}=\boldsymbol{O}_{M \times K} .
$$

Typically,

$$
M \ll K,
$$

can be assumed in the practical criteria of spectrally precoding schemes (Xu and Chen, 2009; van de Beek, 2009, 2010; Ma et al., 2011). Among various designs for the spectral precoder of $\boldsymbol{G}$ to satisfy $\boldsymbol{A} \boldsymbol{G}=\boldsymbol{O}$, SO precoding is one of excellent implementations with an outstanding BER performance very close to that of the plain OFDM. The precoder in the SO precoding is determined as:

$$
\boldsymbol{G}=\boldsymbol{G}_{\mathrm{o}}=\boldsymbol{Q} \tilde{\boldsymbol{E}}_{M} \in \mathbb{C}^{D \times K}
$$

where $\boldsymbol{Q} \in \mathbb{C}^{K \times K}$ is unitary, $\tilde{\boldsymbol{E}}_{M}=\left[\boldsymbol{O}_{D \times M} \boldsymbol{I}_{D}\right]^{T} \in \mathbb{R}^{K \times D}$ and $\tilde{\boldsymbol{E}}_{M}^{T} \tilde{\boldsymbol{E}}_{M}=\boldsymbol{I}_{D}$. In other words, $\boldsymbol{G}_{\mathrm{o}}$ is a submatrix of $\boldsymbol{Q}$ composed the last $D$ columns. To satisfy $\boldsymbol{A} \boldsymbol{G}_{\mathrm{o}}, \boldsymbol{Q}$ is obtained via orthonormalization (Golub and Van Loan, 1996) on $\boldsymbol{A}$ as follows:

$$
\boldsymbol{A} \boldsymbol{Q}=\boldsymbol{R}^{\mathrm{H}}=\left[\begin{array}{ll}
\boldsymbol{R}_{M}^{\mathrm{H}} & \boldsymbol{O}_{M \times D}
\end{array}\right]
$$

where $\boldsymbol{R} \in \mathbb{C}^{M \times K}$ and $\boldsymbol{R}_{M} \in \mathbb{C}^{M \times M}$. The orthonormalization in (8) can be implemented by an arbitrary algorithm. In this paper, SO precoder denotes a class of spectral precoder satisfying

$$
\boldsymbol{G}^{\mathrm{H}} \boldsymbol{G}=\boldsymbol{I}_{D} .
$$

It allows the receiver to perform a postdecoding by $\boldsymbol{G}^{\mathrm{H}}$ to invert the precoding by $\boldsymbol{G}$. Trivially, (7) is an SO precoder because (9) follows from the orthonormality of $\boldsymbol{Q}$.

\section{Proposed Scheme}

This paper proposes an effective precoding scheme to achieve not only PAPR reduction but also OOBE suppression. The precoder in the proposed method is determined as follows:

$$
\boldsymbol{G}=\boldsymbol{G}_{\mathrm{o}} \boldsymbol{W}_{D} \in \mathbb{C}^{K \times D} .
$$

The precoder in (10) is also an SO precoder due to the orthonormality of $\boldsymbol{G}_{\mathrm{o}}$ and $\boldsymbol{W}_{\boldsymbol{D}}$. With this precoder, the proposed precoding is interpreted as

$$
\overline{\boldsymbol{d}}_{i}=\boldsymbol{G}_{\mathrm{o}} \tilde{\boldsymbol{d}}_{i} \in \mathbb{C}^{K \times 1},
$$

where $\tilde{\boldsymbol{d}}_{i}=\boldsymbol{W}_{D} \boldsymbol{d}_{i}$ denotes a precoded symbol equivalent to that in the DFT-s-OFDM. In other words, the DFT-precoding and SO precoding are concatenated as inner and 


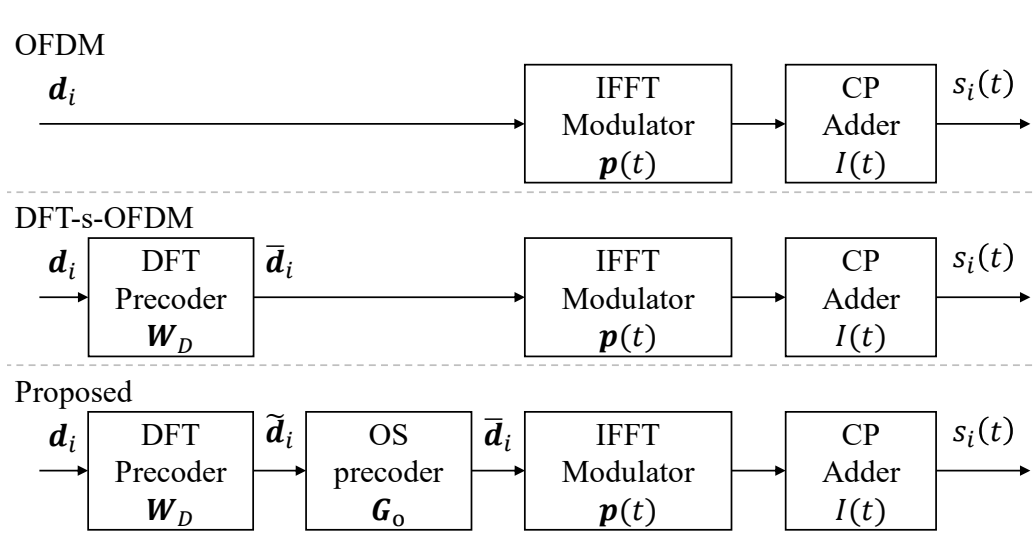

Fig. 1: Proposed Transmitter.

outer linear precoding in the proposed method. Fig. 1 shows the proposed transmitter diagram compared with the usual OFDM and DFT-s-OFDM counterparts.

At the receiver, the proposed postdecoding is implemented in the reverse order. The outer postdecoding is determined to invert the precoding by $\boldsymbol{G}_{\mathrm{o}}$ as

$$
\tilde{\boldsymbol{r}}_{i}=\boldsymbol{G}_{\mathrm{o}}^{\mathrm{H}} \overline{\boldsymbol{r}}_{i} \in \mathbb{C}^{D \times 1}
$$

where $\overline{\boldsymbol{r}}_{i}$ denotes the $i$-th received symbol after some FEQ like zero-forcing (ZF) one thanks to the CP. Then, the inner postdecoding by $\boldsymbol{r}_{i}=\boldsymbol{W}_{D}^{\mathrm{H}} \tilde{\boldsymbol{r}}_{i}$ is performed to cancel the DFT-precoding. As a result, $\boldsymbol{r}_{i}$ is a demodulated symbol on $\boldsymbol{d}_{i}$; if $\overline{\boldsymbol{r}}_{i}=\overline{\boldsymbol{d}}_{i}, \tilde{\boldsymbol{r}}_{i}=\tilde{\boldsymbol{d}}_{i}$ and $\boldsymbol{r}_{i}=\boldsymbol{d}_{i}$ is satisfied. The DFTs for $\tilde{\boldsymbol{d}}_{i}$ and $\boldsymbol{r}_{i}$ can be efficiently implemented in the same way as those in the DFT-s-OFDM by the FFT algorithm with a logarithmic complexity. Hence, the computational complexity with $\boldsymbol{G}_{\mathrm{o}}$ for $\overline{\boldsymbol{d}}_{i}$ and $\tilde{\boldsymbol{r}}_{i}$ is important to practically implement the proposed method.

A direct use of $\boldsymbol{G}_{\mathrm{o}} \in \mathbb{C}^{K \times D}$ results in the complexity of $O\left(K^{2}\right)$ (van de Beek, 2010), which cause high implementation complexity for the SO precoding schemes. To solve this problem, basis-kernel representations (Sun and Bischof, 1995) for orthogonal factors $\boldsymbol{Q}$ can be utilized to efficiently construct SO precoders $\boldsymbol{G}_{\mathrm{o}}$; the complexity is much reduced from $O\left(K^{2}\right)$ to $O(K M)$, see (Clarkson, 2017; Kawasaki et al., 2018, 2019) Motivated by the results in (Kawasaki et al., 2018), the proposed method determines an SO precoder via the compact WY representation, which is easy to compute among the basis-kernel representations and considerably numerical stable. The compact WY representation of $\boldsymbol{Q}$ is obtained as (Schreiber and Van Loan, 1989)

$$
Q=I_{K}-Y T Y^{\mathrm{H}},
$$

where $\boldsymbol{Y} \in \mathbb{C}^{K \times M}$ is a unit lower trapezoidal matrix, $\boldsymbol{T} \in \mathbb{C}^{M \times M}$ is an upper triangular matrix with real-valued diagonal elements and $\boldsymbol{Y}^{\mathrm{H}} \boldsymbol{Y}=\boldsymbol{T}^{-H}+\boldsymbol{T}^{-1}$ is satisfied (thus, $\boldsymbol{Q}$ becomes unitary). When $\boldsymbol{Q}$ is as in (13), $\boldsymbol{R}$ in (8) is an upper triangular matrix and $\boldsymbol{A}=\boldsymbol{Q} \boldsymbol{R}$ denotes a $\mathrm{QR}$ decomposition of $\boldsymbol{A}$. 
With (13), (11) and (12) can be rewritten, respectively, as

$$
\overline{\boldsymbol{d}}_{i}=\tilde{\boldsymbol{E}}_{M} \tilde{\boldsymbol{d}}_{i}-\boldsymbol{Y} \boldsymbol{T}\left(\tilde{\boldsymbol{E}}_{M}^{T} \boldsymbol{Y}\right)^{\mathrm{H}} \tilde{\boldsymbol{d}}_{i},
$$

and

$$
\tilde{\boldsymbol{r}}_{i}=\tilde{\boldsymbol{E}}_{M}^{T} \overline{\boldsymbol{r}}_{i}-\left(\tilde{\boldsymbol{E}}_{M}^{T} \boldsymbol{Y}\right) \boldsymbol{T}^{\mathrm{H}} \boldsymbol{Y}^{\mathrm{H}} \overline{\boldsymbol{r}}_{i} .
$$

The computational complexities of (14) and (15) are in $O(K M)$ and also equivalent. With respect to complex multiplication, the amount is $N_{O P}=(K+D) M=(2 K-$ $M) M \approx 2 K M$, which matches to the same level at the FFT for OFDM or the DFT-precoding (Kawasaki et al., 2018). The proposed method has certain feasibility since the precoding and postdecoding with $\boldsymbol{G}_{\mathrm{o}}$ can be efficiently performed in addition to the counterparts by $\boldsymbol{W}_{D}$.

In the following subsections, we analyze the performance of the proposed method in terms of OOBE and PAPR theoretically.

\subsection{OOBE Suppression}

We show that the proposed method can suppress the OOBE of the OFDM signals. For (2) and (11), the transmitted OFDM symbols in the proposed method is written as

$$
s_{i}(t)=\boldsymbol{p}^{T}(t) \overline{\boldsymbol{d}}_{i}=\boldsymbol{p}^{T}(t) \boldsymbol{G}_{\mathrm{o}} \boldsymbol{G}_{\mathrm{o}} \boldsymbol{d}_{i}=\boldsymbol{p}^{T}(t) \boldsymbol{G}_{\mathrm{o}} \tilde{\boldsymbol{d}}_{i} .
$$

The PSD of (16) is derived as

$$
\begin{aligned}
P(f) & =\frac{1}{T} \boldsymbol{a}^{T}(f) \boldsymbol{G}_{\mathrm{o}} \boldsymbol{W}_{D} \mathbb{E}\left\{\boldsymbol{d}_{i} \boldsymbol{d}_{i}\right\} \boldsymbol{G}_{\mathrm{o}}^{\mathrm{H}} \boldsymbol{W}_{D}^{\mathrm{H}} \boldsymbol{a}^{*}(f) \\
& =\frac{1}{T} \boldsymbol{a}^{T}(f) \boldsymbol{G}_{\mathrm{o}} \boldsymbol{G}_{\mathrm{o}}^{\mathrm{H}} \boldsymbol{a}^{*}(f)=\frac{1}{T}\left\|\boldsymbol{G}_{\mathrm{o}}^{T} \boldsymbol{a}(f)\right\|_{2}^{2},
\end{aligned}
$$

where $\boldsymbol{a}(f)$ is the Fourier transform of $\boldsymbol{p}(t)$. Eq. (17)) is invariant to $\boldsymbol{W}_{D}$ and identical to the counterpart by $\boldsymbol{G}=\boldsymbol{G}_{\mathrm{o}}$, that is, of the means of (van de Beek, 2010). Therefore, the proposed method can achieve a strong OOBE suppression same as the conventional schemes with spectral precoding.

\subsection{PAPR Reduction}

We analyze the PAPR reduction of the proposed method. Substitute $\boldsymbol{P}=\boldsymbol{I}_{K}-\boldsymbol{Q}$ to take $\boldsymbol{G}_{\mathrm{o}}=\boldsymbol{Q} \tilde{\boldsymbol{E}}_{M}=\tilde{\boldsymbol{E}}_{M}-\boldsymbol{P} \tilde{\boldsymbol{E}}_{M}$. We can rewrite (16) into

$$
s_{i}(t)=\boldsymbol{p}^{T}(t) \tilde{\boldsymbol{E}}_{M} \boldsymbol{W}_{D} \boldsymbol{d}_{i}-\boldsymbol{p}^{T}(t) \boldsymbol{P} \tilde{\boldsymbol{E}}_{M} \boldsymbol{W}_{D} \boldsymbol{d}_{i} .
$$

Because of $\boldsymbol{p}^{T}(t) \tilde{\boldsymbol{E}}_{M}=\left[p_{k_{M}}, p_{k_{M+1}}, \ldots, p_{k_{K-1}}\right]^{T}$, the first term in (18) coincides an usual DFT-s-OFDM symbol with subcarrier mapping $\mathcal{D}=\left\{k_{M}, k_{M+1}, \ldots, k_{K-1}\right\}$ and 
$|\mathcal{D}|=D$. Accordingly, in the aspect of power we define a relative error of the symbol (18) to the DFT-s-OFDM symbol in the first term as follows:

$$
e=\frac{\mathbb{E}\left\{\left\|\boldsymbol{p}^{T}(t) \boldsymbol{P} \tilde{\boldsymbol{E}}_{M} \boldsymbol{W}_{D} \boldsymbol{d}_{i}\right\|_{2}^{2}\right\}}{\mathbb{E}\left\{\left\|\boldsymbol{p}^{T}(t) \tilde{\boldsymbol{E}}_{M} \boldsymbol{W}_{D} \boldsymbol{d}_{i}\right\|_{2}^{2}\right\}}=\frac{\left\|\boldsymbol{P} \tilde{\boldsymbol{E}}_{M}\right\|_{F}^{2}}{\left\|\boldsymbol{I}_{D}\right\|_{F}^{2}}=\frac{\left\|\boldsymbol{P} \tilde{\boldsymbol{E}}_{M}\right\|_{F}^{2}}{D} .
$$

We remark that $e$ in (19) is invariant to $t$. Furthermore, (19)) can reduce to

$$
e=\frac{2 \operatorname{Re} \operatorname{Tr}\left\{\tilde{\boldsymbol{E}}_{M}^{T} \boldsymbol{P} \tilde{\boldsymbol{E}}_{M}\right\}}{D},
$$

because $\left\|\boldsymbol{P} \tilde{\boldsymbol{E}}_{M}\right\|_{F}^{2}=\operatorname{Tr}\left\{\tilde{\boldsymbol{E}}_{M}^{T} \boldsymbol{P}^{\mathrm{H}} \boldsymbol{P} \tilde{\boldsymbol{E}}_{M}\right\}=\operatorname{Tr}\left\{\tilde{\boldsymbol{E}}_{M}^{T}\left(\boldsymbol{P}^{\mathrm{H}}+\boldsymbol{P}\right) \tilde{\boldsymbol{E}}_{M}\right\}=2 \operatorname{Re} \operatorname{Tr}\left\{\tilde{\boldsymbol{E}}_{M}^{T} \boldsymbol{P} \tilde{\boldsymbol{E}}_{M}\right\}$ follows from $\boldsymbol{Q}^{\mathrm{H}} \boldsymbol{Q}=\boldsymbol{I}_{K}$ and thus $\boldsymbol{P}^{\mathrm{H}} \boldsymbol{P}=\boldsymbol{P}+\boldsymbol{P}^{\mathrm{H}}$.

Next, we give some inequalities on $e$. From $\boldsymbol{I}_{K}=\left[\begin{array}{ll}\boldsymbol{E}_{M} & \tilde{\boldsymbol{E}}_{M}\end{array}\right]$, we have $\|\boldsymbol{P}\|_{F}^{2}=$ $\left\|\boldsymbol{P} \boldsymbol{E}_{M}\right\|_{F}^{2}+\left\|\boldsymbol{P} \tilde{\boldsymbol{E}}_{M}\right\|_{F}^{2}$ to take $\left\|\boldsymbol{P} \tilde{\boldsymbol{E}}_{M}\right\|_{F}^{2} \leq\|\boldsymbol{P}\|_{F}^{2}$. In addition, $\|\boldsymbol{P}\|_{F}^{2}=\operatorname{Tr}\left\{\boldsymbol{P}^{\mathrm{H}} \boldsymbol{P}\right\}=$ $\operatorname{Tr}\left\{\boldsymbol{P}^{\mathrm{H}}+\boldsymbol{P}\right\}=2 \operatorname{Re} \operatorname{Tr}\{\boldsymbol{P}\}$ holds. Therefore, $\operatorname{Re} \operatorname{Tr}\left\{\tilde{\boldsymbol{E}}_{M}^{\top} \boldsymbol{P} \tilde{\boldsymbol{E}}_{M}\right\} \leq \operatorname{Re} \operatorname{Tr}\{\boldsymbol{P}\}$ is shown. All the non-zero eigenvalues of $\boldsymbol{P}$ (its number coincides with $\operatorname{rank}\{\boldsymbol{P}\}$ ) exist on unit circle in complex plane because $\boldsymbol{Q}=\boldsymbol{I}_{K}-\boldsymbol{P}$ is unitary with the eigenvalues all equal to 1 in absolute value. That is, any non-zero eigenvalue of $\boldsymbol{P}$ has a real part between 0 and 2 . Thus, we get

$$
\operatorname{Re} \operatorname{Tr}\left\{\tilde{\boldsymbol{E}}_{M}^{\top} \boldsymbol{P} \tilde{\boldsymbol{E}}_{M}\right\} \leq \operatorname{Re} \operatorname{Tr}\{\boldsymbol{P}\} \leq 2 \operatorname{rank}\{\boldsymbol{P}\},
$$

and thereby

$$
e \leq \frac{2 \operatorname{Re} \operatorname{Tr}\{\boldsymbol{P}\}}{D} \leq \frac{4 \operatorname{rank}\{\boldsymbol{P}\}}{D} .
$$

Note that the above discussion is consistent with $\boldsymbol{Q}$ obtained by an arbitrary orthonormalization method.

In the case of the compact WY representation, $\operatorname{rank}\{\boldsymbol{P}\}=\operatorname{rank}\left\{\boldsymbol{Y} \boldsymbol{T} \boldsymbol{Y}^{\mathrm{H}}\right\} \leq$ $\operatorname{rank}\{\boldsymbol{Y}\} \leq M$ holds further. It deduces the following inequality for (19):

$$
e_{c w y}=\frac{2 \operatorname{Re} \operatorname{Tr}\left\{\tilde{\boldsymbol{E}}_{M}^{\top} \boldsymbol{Y} \boldsymbol{T} \boldsymbol{Y}^{\mathrm{H}} \tilde{\boldsymbol{E}}_{M}\right\}}{D} \leq \frac{4 M}{D} .
$$

$e_{c w y}$ in (23) is small under (6).

\section{Numerical Evaluation}

Numerical experiments were conducted to evaluate the performance of the proposed method based on the parameters of the 3GPP specifications (3GPP, 2016a) shown in TABLE 1 .

For the numerical experiments, $\boldsymbol{G}_{\mathrm{o}}$ must be characterized by a specific $\boldsymbol{A}$ for the orthogonally precoded schemes. We determined it based on the spectrum sculpting criterion (van de Beek, 2009, 2010) as a concise instance. In this criterion, $\boldsymbol{A}=\left[\boldsymbol{a}\left(f_{0}\right) \boldsymbol{a}\left(f_{1}\right) \ldots \boldsymbol{a}\left(f_{M-1}\right)\right]^{T} \in \mathbb{C}^{M \times K}\left\{\overline{\boldsymbol{d}}_{i}\right\}$ are constituted to satisfy 
Table 1: Numerical experimental conditions (3GPP, 2016a).

\begin{tabular}{c||ccc}
\hline Parameter & Value A & Value B & Value C \\
\hline OFDM symbol duration $T_{\mathrm{s}}$ & \multicolumn{3}{c}{$1 / 15 \mathrm{~ms}$} \\
CP length $T_{\mathrm{g}}$ & \multicolumn{3}{|c}{$9 T_{\mathrm{s}} / 128$} \\
Modulation level & \multicolumn{3}{|c}{$16-\mathrm{QAM}$} \\
Average transmit power & \multicolumn{3}{|c}{$23 \mathrm{dBm}$} \\
Subcarrier mapping $\mathcal{K}$ & $\{-K / 2,-K / 2+1, \ldots, K / 2-1\}$ \\
Number of information symbols $D$ & 72 & 180 & 600 \\
Transmission bandwidth & $1.4 \mathrm{MHz}$ & $3 \mathrm{MHz}$ & $10 \mathrm{MHz}$ \\
\hline
\end{tabular}

$P(f)=\boldsymbol{a}^{\top}\left(f_{m}\right) \boldsymbol{d}_{i}=0$ in (17) at the well-chosen frequencies $f_{m}$ collected in $\mathcal{M}=$ $\left\{f_{0}, f_{1}, \ldots, f_{M-1}\right\}$. Further we chose the $M=8$ frequencies for $\mathcal{M}$.

In the experimental results, the labels of "OFDM", "DFT-s-OFDM", "RC windowed", "” and "Proposal" correspond to the plain OFDM, usual DFT-s-OFDM, windowing method (Weiss et al., 2004), the means of (Clarkson, 2017), and proposed method, respectively. The RC windowed method was characterized as the DFT-s-OFDM with the raised-cosine window function with roll-off factor $\beta=$ $T_{\mathrm{g}} / T$. The conventional method is another orthogonally precoded scheme where the DFT-precoding and SO precoding are concatenated in reverse order to the proposed method; $\boldsymbol{G}=\boldsymbol{W}_{K} \tilde{\boldsymbol{G}}_{\mathrm{o}}$ is used where (5) is replaced with $\tilde{\boldsymbol{A}} \tilde{\boldsymbol{G}}_{\mathrm{o}}=\boldsymbol{O}$ and $\tilde{\boldsymbol{A}}=\boldsymbol{A} \boldsymbol{W}_{K}$, and $\tilde{\boldsymbol{G}}_{\mathrm{o}}^{\mathrm{H}}$ is obtained via another basis-kernel representation referred to as block Householder reflector (Schreiber and Parlett, 1987). On the other hand, In the conventional method has no theoretical guarantees for the PAPR reduction corresponding to (23).

\subsection{PSD and BER Performance}

The PSD and BER characteristics of the proposed method were measured. Fig.2 and Fig. 3 show the results of the PSD and BER, respectively.

In the PSD results of Fig. 2 where the signals were 8-times oversampled, the dotted lines denote the spectral mask of $55 \mathrm{~dB}$ at the transmission band-edges. The results of the OFDM and conventional method were omitted because they are identical to that of the DFT-s-OFDM and proposed method, respectively.

For the measurement of the BER performance, the TDL-C channel model characterized by the normal-delay profile for Urban Macro system using $2 \mathrm{GHz}$ band and the maximum Doppler frequency $5.56 \mathrm{~Hz}$ (3GPP, 2016b) was used to constitute experimental fading channels. At the receiver, the ZF FEQ after the perfect estimation of the channel characteristics was assumed. The theoretical curve for OFDM case in an AWGN channel were also plotted as a reference. In the AWGN channel, it is theoretically shown that all the methods have the same BER performance as the OFDM.

From the results of Fig.2 and Fig.3, the RC windowed method cannot satisfy the spectral mask at $D=72$ and $D=180$, and its BER performance was degraded compared with that of the DFT-s-OFDM and indicated a performance floor in the fading channels due to the reduced effective length of the CP. In contrast, the 


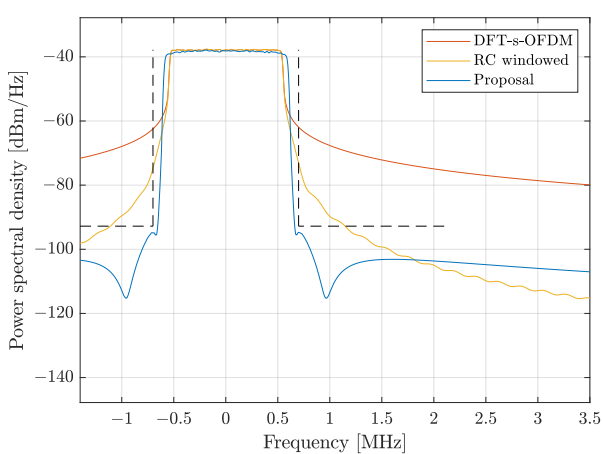

(a) Value $\mathrm{A}$ and $\mathcal{M}=\{ \pm 660 \pm 1, \pm 960 \pm 1\}-7.5 \mathrm{kHz}$.

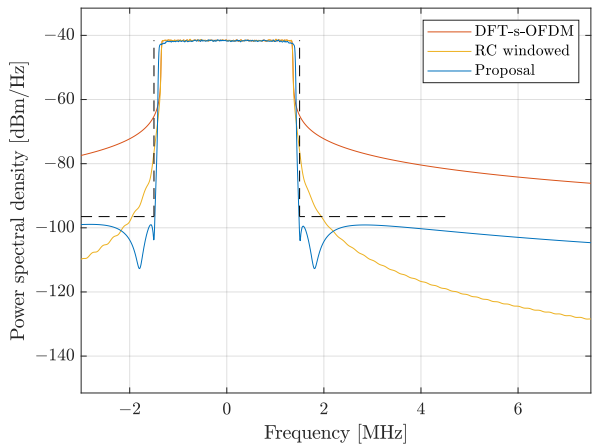

(b) Value $\mathrm{B}$ and $\mathcal{M}=\{ \pm 1500 \pm 1, \pm 1800 \pm 1\}-7.5 \mathrm{kHz}$.

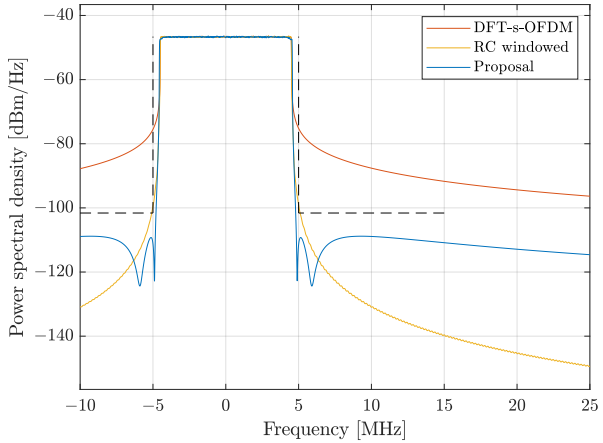

(c) Value $\mathrm{C}$ and $\mathcal{M}=\{ \pm 4900 \pm 1, \pm 5900 \pm 1\}-7.5 \mathrm{kHz}$

Fig. 2: PSD results.

proposed method a strong OOBE suppression to render the PSD results less than the DFT-s-OFDM by about $25 \mathrm{~dB}$ at band-edges and meet the spectral mask without BER degradation for all the values of $D$. As a result, it is verified that the proposed method can suppress the OOBE of the OFDM signals strongly as keeping an ideal BER performance. 


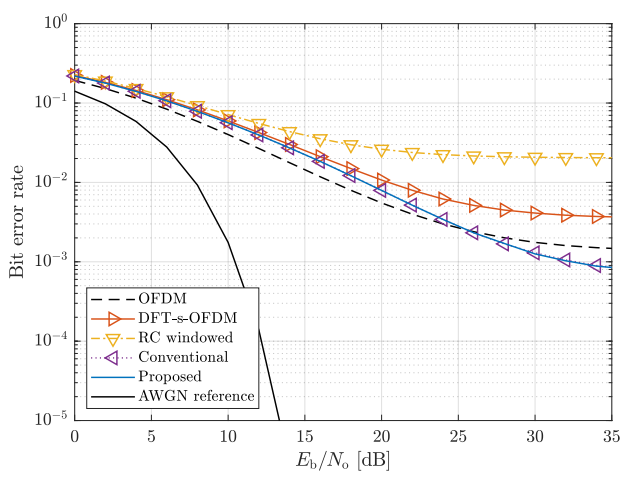

(a) Signals of Fig.2(a).

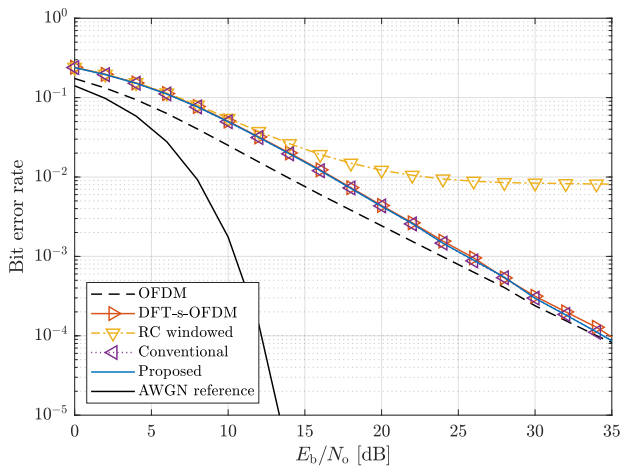

(b) Signals of Fig.2(b).

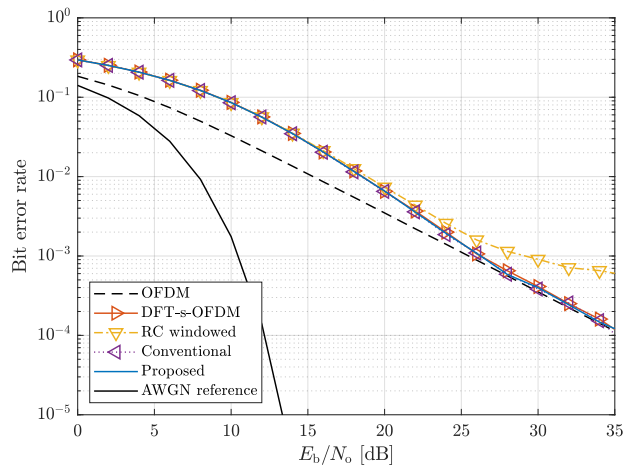

(c) Signals of Fig.2(c).

Fig. 3: BER results.

\subsection{PAPR Performance}

The PAPR characteristics were compared using the same parameters of Fig.2 except for the modulation level of QPSK. Fig.4 shows the measured complementary cumulative distribution function (CCDF) through 16-times oversampling for OFDM 


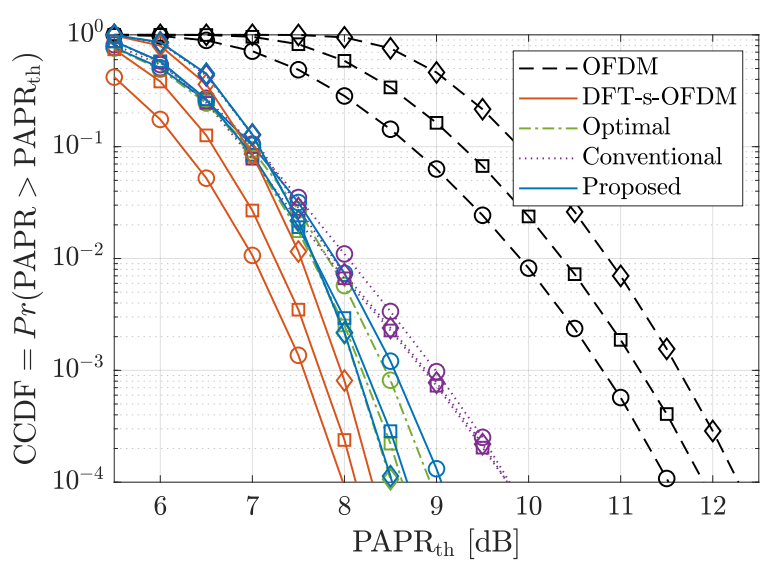

(a) QPSK, $D=72(\bigcirc), D=180(\square), D=600(\diamond)$

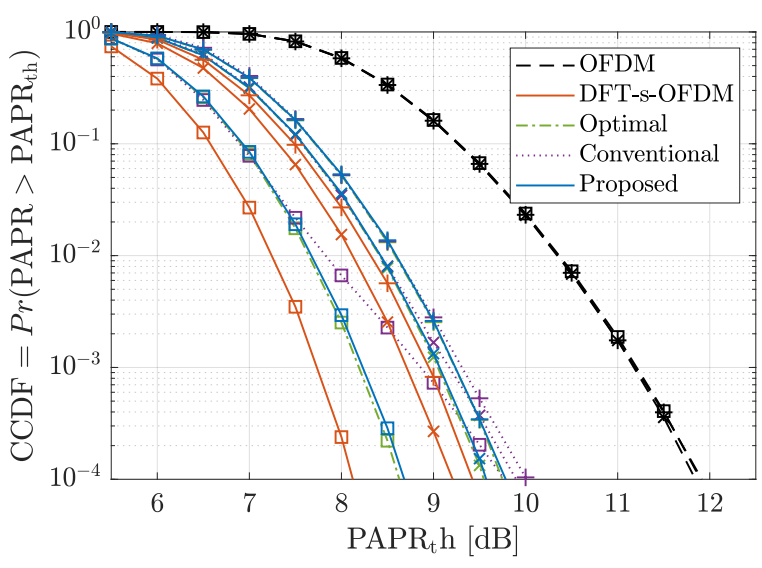

(b) $(D, M)=(180,8)$, QPSK $(\square), 16-\mathrm{QAM}(\times), 64-\mathrm{QAM}(+)$

Fig. 4: PAPR characteristics of the proposed method.

symbols. As a reference, the results using the SO precoder to minimize $e$ in (19) (Ma et al., 2011) were also shown using the label of "Optimal". The values of (19) is evaluated as $14 \%, 5.9 \%$ and $2.1 \%$ in the proposed method for $D=72, D=180$ and $D=600$, respectively. They are much near to the counterparts evaluated as $13 \%$, $5.7 \%$ and $2.0 \%$ in the optimal method. The CCDF was defined on the probability of PAPR $=\max \left\{\left|s_{i}(t)\right|^{2}\right\} / D$ for $-T_{\mathrm{g}} \leq t<T_{\mathrm{s}}$ considering that $\mathbb{E}\left\{\left|s_{i}(t)^{2}\right|\right\}=D$ is satisfied in all the methods. In addition, the results of the RC windowed method were omitted because it is identical to that of the usual DFT-s-OFDM theoretically. From the results, the proposed method was outstanding over the OFDM. The performance gain was roughly $2 \mathrm{~dB}$ at CCDF of $10^{-4}$ in Fig.4(a). Although the 
proposed method was also worse than the DFT-s-OFDM, the performance loss is relatively marginal compared with the conventional method. For example, the loss of the proposed method was limited within about $1 \mathrm{~dB}$ in the case of $D=72$ in Fig.4(a) while the counterpart of the conventional method reached to roughly $2 \mathrm{~dB}$. The DFT-s-OFDM signals have high PAPR characteristics for a large $D$ (Myung et al., 2006), as verified in Fig.4(a). On the other hand, the PAPR performance was improved in the proposed method as $D$ increases. It suggests that with a large value of $D$, the decrease of (19) is dominant and the difference between the proposed method and DFT-s-OFDM decreases in terms of PAPR performance. Similarly, the performance difference between the proposed method and DFT-s-OFDM became small when a high modulation level like 64-QAM as shown in Fig.4(b) with different modulation levels of QPSK, 16-QAM and 64-QAM for $D=180$. It is also shown that the performance of the proposed method is very close to that the optimal method in every case. The precoder in the optimal method, on the other hand, is not efficiently represented in (Ma et al., 2011) in contrast to the conventional and proposed methods, and thus heavy computational complexities are caused by the direct use of $\boldsymbol{G}_{o} \in \mathbb{C}^{D \times K}$ as previously discussed. Therefore, the proposed method has a good practicality to achieve low PAPR characteristics by low complexity.

\section{Conclusion}

This paper proposes an affective linear precoding scheme to resolve the major problems of the OFDM systems like high PAPR and high OOBE. Its effectiveness is verified in the theoretical ways. The numerical experiments also show that the proposed method can achieve a strong OOBE suppression as keeping an ideal BER performance regardless of transmission bandwidth. It is also presented that the performance trajectory on PAPR is similar to that of the usual DFT-s-OFDM. The key advantage of the proposed method compared to the plain OFDM scheme is that a strong OOBE suppression by about $25 \mathrm{~dB}$ at band-edges, a practical PAPR reduction by roughly $2 \mathrm{~dB}$ better, no BER degradation, and a sufficiently practical computational complexity matching to that of the FFT can be simultaneously achieved. It suggests that the proposed method can resolve the problems with a minimal implantation cost in the conventional OFDM systems already equipped with precoding structure.

\section{References}

3GPP (2016a) Evolved Universal Terrestrial Radio Access (E-UTRA); Physical channels and modulation. Technical Specifications (TS) 36.211 V13.2.0, 3rd Generation Partnership Project (3GPP)

3GPP (2016b) Study on channel model for frequency spectrum above $6 \mathrm{GHz}$. Technical Report (TR) 38.900 V.14.2.0, 3rd Generation Partnership Project (3GPP)

3GPP (2018) General aspects for User Equipment (UE) Radio Frequency (RF) for NR. Technical Report (TR) 38.817-01 V.15.1.0, 3rd Generation Partnership Project (3GPP) 
van de Beek J (2009) Sculpting the multicarrier spectrum: a novel projection precoder. IEEE Commun Lett 13(12)

van de Beek J (2010) Orthogonal Multiplexing in a Subspace of Frequency Well-Localized Signals. IEEE Commun Lett 14(10):882-884

van de Beek J, Berggren F (2009) $N$-continuous OFDM. IEEE Commun Lett 13(1):1-3

Brandes S, Cosovic I, Schnell M (2006) Reduction of Out-of-Band Radiation in OFDM Systems by Insertion of Cancellation Carriers. IEEE Commun Lett $10(6): 420-422$

Chung CD (2006a) Correlatively coded OFDM. IEEE Trans Wireless Commun 5(8):2044-2049

Chung CD (2006b) Spectrally precoded OFDM. IEEE Trans Commun 54(12):2173-2185

Chung CD (2008) Spectral precoding for rectangularly pulsed OFDM. IEEE Trans Commun 56(9):1498-1510

Clarkson IVL (2017) Orthogonal Precoding for Sidelobe Suppression in DFT-Based Systems using Block Reflectors. In: Proc. IEEE ICASSP, pp 3709-3713

Golub GH, Van Loan CF (1996) Matrix computations. Johns Hopkins University, Press, Baltimore, MD, USA pp 374-426

Han SH, Lee JH (2005) An Overview of Peak-to-Average Power Ratio Reduction Techniques for Multicarrier Transmission. IEEE Wireless Commun 12(2):56-65

Hussain K, Lojo A, López-Valcarce R (2019) Flexible Spectral Precoding for Sidelobe Suppression in OFDM Systems. In: IEEE ICASSP, pp 4789-4793, DOI 10.1109/ICASSP.2019.8683162

Kawasaki H, Ishizu K, Kojima F (2018) Computationally Efficient Orthogonal Precoding for Sidelobe Suppression of OFDM Signals. In: Proc. IEEE ICC, IEEE, pp 1-6

Kawasaki H, Matsumura T, Ishizu K, Kojima F (2019) Low-complexity Orthogonal $N$-continuous Precoding for Sidelobe Suppression of OFDM Signals. In: Proc. IEEE ICC, IEEE, pp 1-6

Ma M, Huang X, Jiao B, Guo YJ (2011) Optimal Orthogonal Precoding for Power Leakage Suppression in DFT-based Systems. IEEE Trans Commun 59(3):844-853

Mizutani K, Matsumura T, Harada H (2019) Comprehensive Performance Evaluation of Universal Time-domain Windowed OFDM-based LTE Downlink System. IEICE Trans Commun E102-B(8):1728-1740

Mohamad M, Nilsson R, van de Beek J (2018) A Novel Transmitter Architecture for Spectrally-Precoded OFDM. IEEE Trans Circuits and Syst I: Regular Papers 65(8):2592-2605

Myung HG, Lim J, Goodman DJ (2006) Single carrier FDMA for uplink wireless transmission. IEEE Vehicular Technology Magazine 1(3):30-38

Pitaval RA, Popović BM, van de Beek J (2017) N-continuous SC-FDMA and its Polarized Transmission and Reception. IEEE Trans on Commun 65(11):4911-4925

Schreiber R, Parlett BN (1987) Block Reflectors: Theory and Computation. SIAM Journal on Numer Anal pp 189-205 
Schreiber R, Van Loan C (1989) A Storage-Efficient WY Representation for Products of Householder Transformations. SIAM J on Sci and Stat Comput 10(1):53-57

Sun X, Bischof C (1995) A Basis-Kernel Representation of Orthogonal Matrices. SIAM journal on matrix analysis and applications 16(4):1184-1196

Weiss T, Hillenbrand J, Krohn A, Jondral FK (2004) Mutual interference in OFDM-based spectrum pooling systems. In: Proc. IEEE VTC 2004-Spring, vol 4, pp 1873-1877

Xu R, Chen M (2009) A Precoding Scheme for DFT-based OFDM to Suppress Sidelobes. IEEE Commun Lett 13(10)

Zhang X, Zhang L, Xiao P, Ma D, Wei J, Xin Y (2018) Mixed Numerologies Interference Analysis and Inter-Numerology Interference Cancellation for Windowed OFDM Systems. IEEE Trans Veh Technol 67(8):7047-7061

Zheng Y, Zhong J, Zhao M, Cai Y (2012) A Precoding Scheme for $N$-continuous OFDM. IEEE Commun Lett 16(12):1937-1940 
Figures

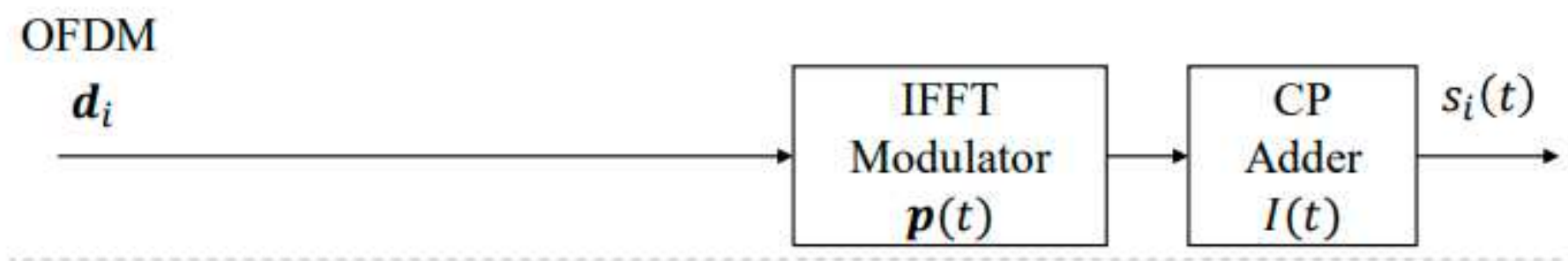

DFT-s-OFDM

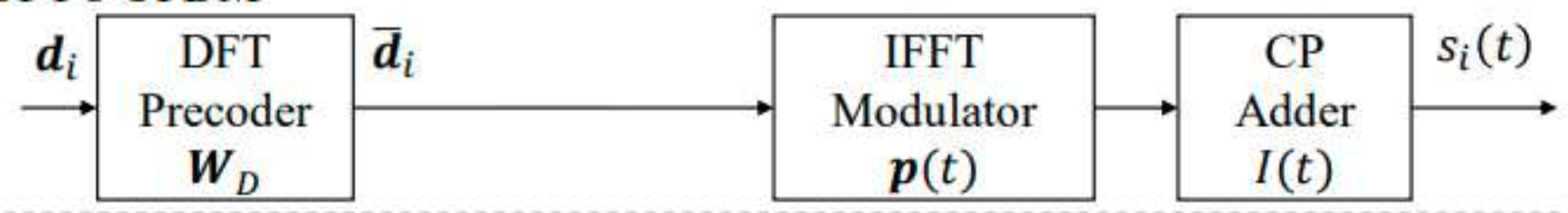

Proposed

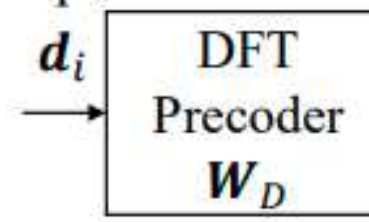

$\stackrel{\widetilde{\boldsymbol{d}}_{i}}{\longrightarrow} \begin{gathered}\text { OS } \\ \text { precoder } \\ \boldsymbol{G}_{\mathrm{O}}\end{gathered}$

$\stackrel{\overline{\boldsymbol{d}}_{i}}{\longrightarrow} \begin{gathered}\text { IFFT } \\ \text { Modulator } \\ \boldsymbol{p}(t)\end{gathered}$
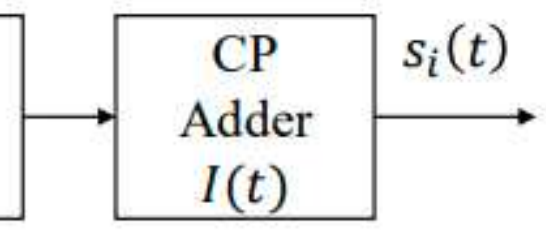

Figure 1

Proposed Transmitter. 


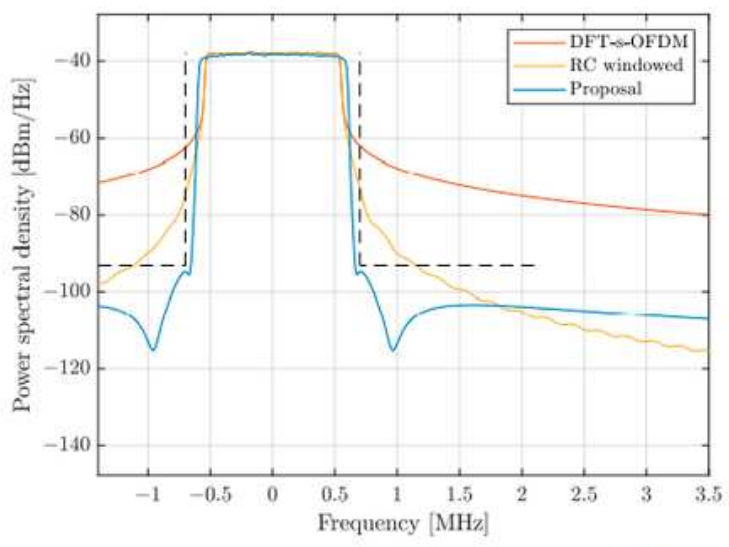

(a) Value $A$ and $\mathcal{M}=\{ \pm 660 \pm 1, \pm 960 \pm 1\}-7.5 \mathrm{kHz}$.

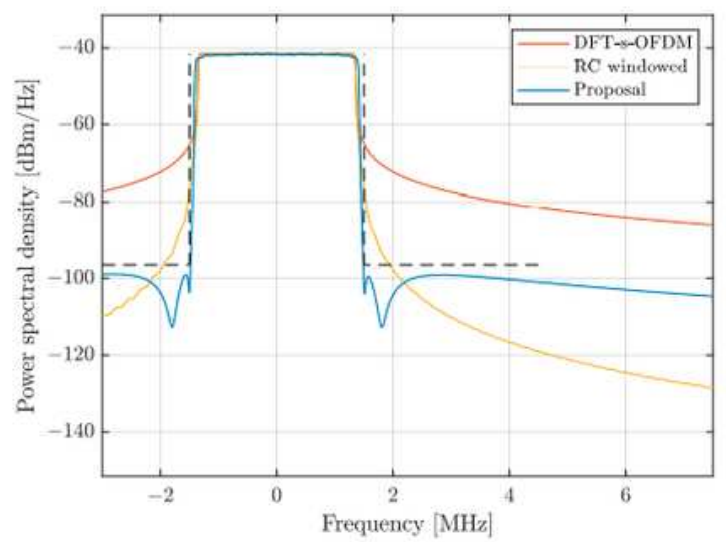

(b) Value $B$ and $\mathcal{M}=\{ \pm 1500 \pm 1, \pm 1800 \pm 1\}-7.5 \mathrm{kHz}$.

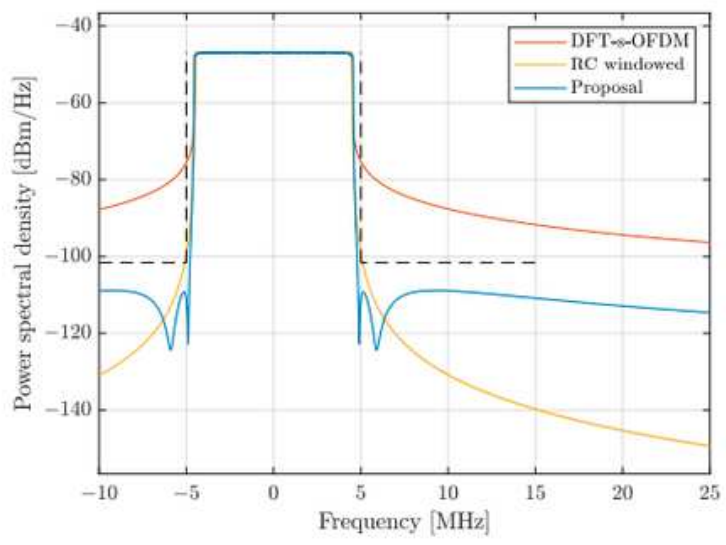

(c) Value $\mathrm{C}$ and $\mathcal{M}=\{ \pm 4900 \pm 1, \pm 5900 \pm 1\}-7.5 \mathrm{kHz}$.

Figure 2

PSD results. 


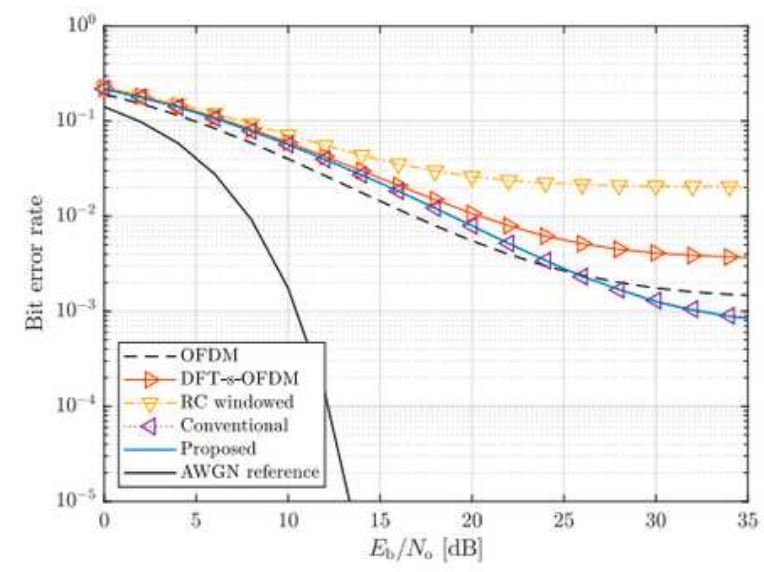

(a) Signals of Fig.2(a).

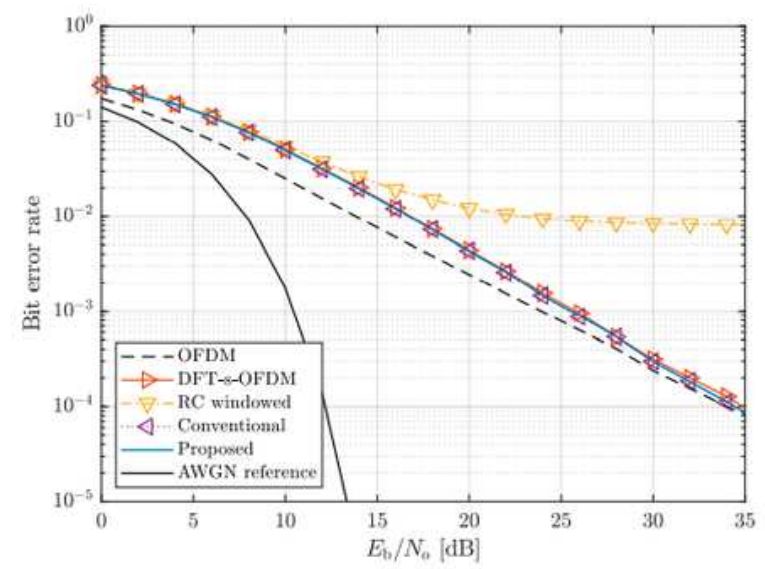

(b) Signals of Fig.2(b).

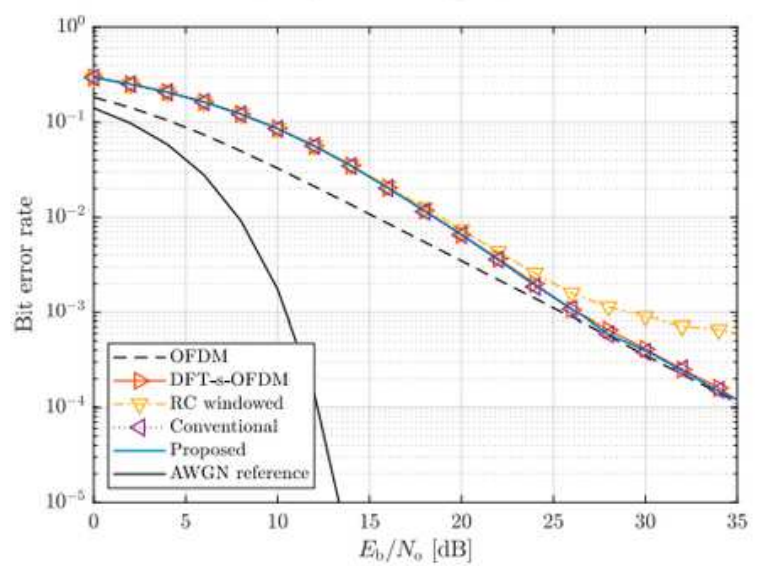

(c) Signals of Fig.2(c).

Figure 3

BER results. 


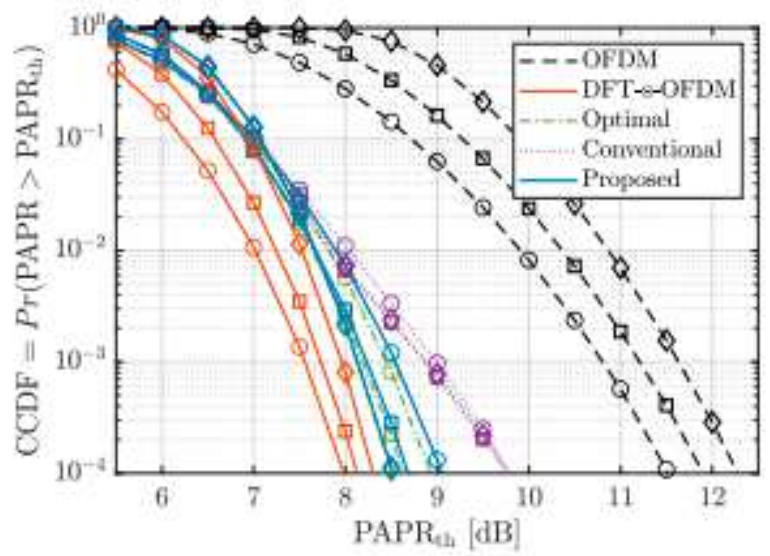

(a) QPSK, $D=72(O), D=180$ (a), $D=600$ ()

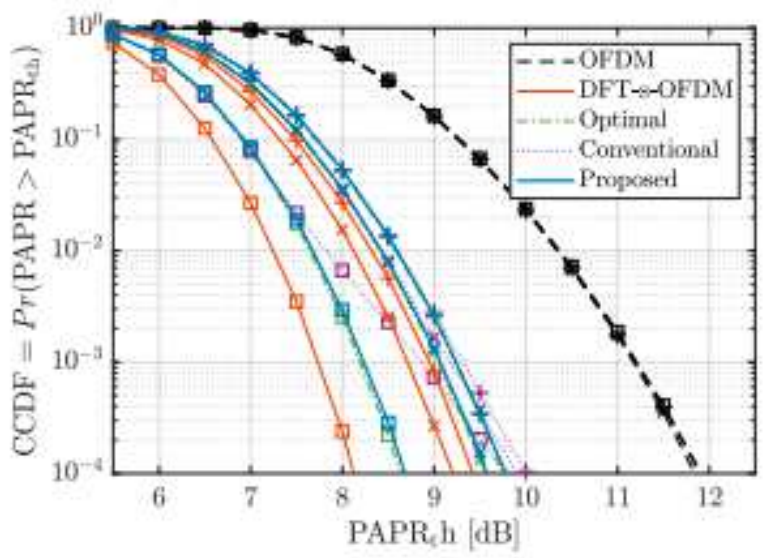

(b) $(D, M)=(180,8)$, QPSK (a), 16-QAM (x), 64-QAM (+)

Figure 4

PAPR characteristics of the proposed method. 